\title{
A PAPR reduction scheme with residue number system for OFDM
}

\author{
Yi Yao ${ }^{*}$, Jianhao Hu and Shang Ma
}

\begin{abstract}
The peak-to-average power (PAPR) is one of the main challenges in multicarrier transmissions. Aiming at reducing the PAPR, we propose a residue number system (RNS)-based OFDM parallel transmission scheme. The key idea of the proposed scheme is to utilize the parallel property of RNS to convert the input signals into the parallel smaller residue signals while utilizing the characteristic of RNS modular operation to effectively limit the output in each residue subchannel after inverse fast Fourier transform, which is smaller than the corresponding modulus. The main contribution of the proposed scheme is to reduce the dynamic range of the transmitted signal without nonlinear distortion so as to reduce the PAPR during the transmission. A generalized performance of the proposed scheme is analyzed in this paper, including the PAPR reduction, the complexity, the transmission bandwidth, etc. Also, an approximate formula to calculate the transmission bandwidth of the proposed scheme is derived, which simplifies design procedure in practice and implies that a minor increase of the dynamic range of RNS will bring comparative improvement of the transmission bandwidth consumption. Theoretical analysis and simulation results demonstrate that the proposed scheme has the ability to achieve desirable PAPR reduction and low computational complexity without nonlinear distortion.
\end{abstract}

\section{Introduction}

Orthogonal frequency division multiplexing (OFDM), known as a multicarrier transmission, divides high-rate serial data streams into a number of parallel lower rate data streams that are transmitted on different subcarriers. The main advantages of OFDM-based systems include robustness to frequency selective fading, high spectral efficiency, low-complexity equalization, etc. [1,2]. However, since the transmitted signal of multicarrier transmission is the sum of data on different subcarriers, the variation of OFDM signal amplitudes is very wide with high peak-to-average power ratio (PAPR). The system performance could be degraded due to high PAPR, which introduces signal distortion when the dynamic range of transmitted signals is larger than the amplifier accommodation. As a consequence, PAPR becomes one of the bottlenecks for OFDM-based systems in practical applications.

These years, great interest has been focused on PAPR reduction [3-10]. In general, these schemes can be

*Correspondence: yaoyi@uestc.edu.cn

National Key Lab of Science and Technology on Communication, University of Electronic Science and Technology of China, Chengdu 611731, China classified into lossy and lossless techniques depending on whether the transmitted signals are distorted or not. Common lossy schemes include clipping, peak windowing, companding transform, etc. Among them, clipping $[5,6]$, which limits parts of the signals over the allowed region, is the simplest and most widely used. However, there are some limitations for these lossy schemes. For example, when the distortion caused by amplitude clipping is serious, it will lead to bit error rate (BER) performance degradation. Lossless schemes include coding [7] and probabilistic scheme [8-10]. Coding scheme selects the codeword that reduces the PAPR for transmission and may address the problem of error control, but it is hard to be adapted to OFDM with a larger number of subcarriers. Probabilistic schemes, such as partial transmit sequence (PTS) and selected mapping (SLM), which are based on decreasing the occurrence probability of peaks instead of avoiding peak of signals, reduce the PAPR effectively without the distortion at the cost of computational complexity and data rate loss due to the use of side information.

Residue number system (RNS), a parallel number system, is based on Chinese remainder theorem (CRT), which divides a large integer into several independent and parallel smaller ones with a specific modulus set. Due to

\section{是 Springer}

(c) 2013 Yao et al. licensee Springer. This is an Open Access article distributed under the terms of the Creative Commons Attribution License (http://creativecommons.org/licenses/by/2.0), which permits unrestricted use, distribution, and reproduction in any medium, provided the original work is properly cited. 
the carry-free and parallel properties, RNS further simplifies the computations by decomposing a problem into a set of parallel, independent residue computations. Thus, RNS has received wide attention in very large scale integration applications. The activities of RNS focus on RNSto-binary conversion, RNS parity check, and RNS scaling scheme [11-13]. Recently, more attention is also paid to RNS in a parallel communication field because of its parallel and fault-tolerant properties [14-19]. For instance, the RNS-based parallel communication scheme like CDMA has been proposed in [14,15], which focused on the system architecture and the BER performance improvement at the receiver. An RNS arithmetic aided frequency-hopping pattern is designed in [16-18], where RNS is invoked as a tool for constructing uniform pilot patterns with limited interference. In [19], we proposed an RNS-based OFDM transmission scheme to reduce the PAPR without nonlinear distortion.

A preliminary study was previously presented in [19], where we concentrated on the system's description and on the PAPR simulation results. In this paper, we extend the performance analysis of the proposed scheme to the PAPR reduction, the complexity, the transmission bandwidth, etc. We will evaluate its performance in comparison with conventional OFDM and PTS-OFDM. The hardware complexity of the proposed scheme will be also discussed. Unlike [14-18], we utilize residue signals to present transmitted signals so as to reduce the PAPR at the transmitter in OFDM multicarrier systems. When an RNS-based transmission scheme is employed in OFDM, one of the big advantages is that the dynamic range of the inverse fast Fourier transform (IFFT) output is limited by the corresponding modulus due to the characteristic of RNS modular operation. The main principle of the proposed scheme is to utilize the parallel property of RNS to divide the original frequency band into $V$ equal portions and to convert the input signals into $V$ smaller residues using the corresponding modulus set. Then, these $V$ residue signals are preformed modulations (in particular, OFDM in this paper) in the corresponding $V$ residue subchannels. Signals of each residue subchannel share the original frequency band through frequency division multiplexing (FDM). Specifically, the value of the corresponding modulus determines the dynamic range of the output in each residue subchannel. When the number of subcarriers is large, the proposed scheme is still able to limit the transmitted signals within a small dynamic range and reduce PAPR without nonlinear distortion. It is demonstrated that the PAPR performance has been improved by more than $5 \mathrm{~dB}$ compared with conventional OFDM. We also find that the proposed scheme outperforms PTS-OFDM in computational complexity.

This paper is organized as follows: In Section 2, the background about OFDM, PAPR, and the properties of
RNS is briefly introduced. The proposed transmission scheme is described, and the performance analysis of PAPR reduction, complexity, and transmission bandwidth is provided in Section 3. The simulation results are given in Section 4, while the conclusions are offered in Section 5.

\section{Background}

\subsection{OFDM and PARR}

OFDM is a transmission scheme which distributes the data over a large number of closely spaced orthogonal subcarriers. The available bandwidth is divided into the orthogonal carriers. The basic structure of OFDM transmission is shown in Figure 1. It multiplexes the data on multiple carriers and transmits them in parallel. Define the input data symbols (i.e., constellation symbols) on the subcarriers as $d_{i}(i=0,1, \ldots, N-1)$, where $N$ is the number of subcarriers. The output after the inverse discrete Fourier transform is $s_{k}$, as shown in (1).

$$
s_{k}=\sum_{i=0}^{N-1} d_{i} \exp \left(j \frac{2 \pi i k}{N}\right),(0 \leq k \leq N-1)
$$

The PAPR of OFDM signals is defined as the ratio between the maximum peak power and the average power [20].

$$
\begin{aligned}
\text { PAPR }= & 10 \log \frac{\max \left\{\left|s_{k}\right|^{2}\right\}}{E\left\{\left|s_{k}\right|^{2}\right\}}(d B) \\
& (k=0,1,2 \ldots, N-1)
\end{aligned}
$$

It is known that the complementary cumulative distribution function (CCDF) is commonly used to denote the probability that the PAPR exceeds a given threshold value $z$, as shown in (3).

$$
P\{\mathrm{PAPR}>z\}=1-\{\mathrm{PAPR} \leq z\}=1-\left(1-e^{-z}\right)^{N}
$$

\subsection{Properties of residue number system}

An RNS is defined by the relatively prime modulus set $m_{v}(v=1,2, \ldots, V)$. Any integer $R$ can be represented in RNS by residue sequence $\left\{r_{1}, r_{2}, \cdots, r_{V}\right\}$, as shown in (4).

$$
r_{v} \equiv R\left(\bmod m_{v}\right)
$$

The number $r_{\nu}$ is said to be the residue of $R$ with respect to $m_{v}$, and we shall usually denote this by $r_{v}=\langle R\rangle_{m_{v}}$. The integers in the range of $\left[0, M_{I}\right)$ can be represented in this

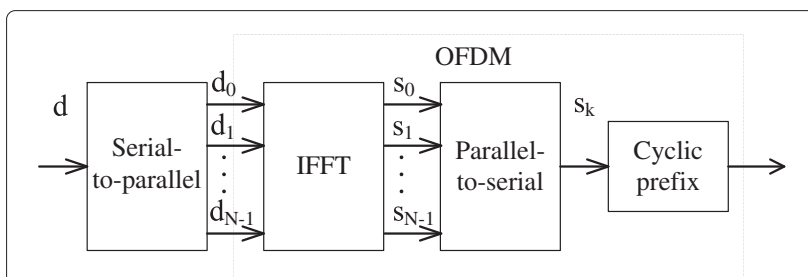

Figure 1 The basic structure of OFDM transmission. 


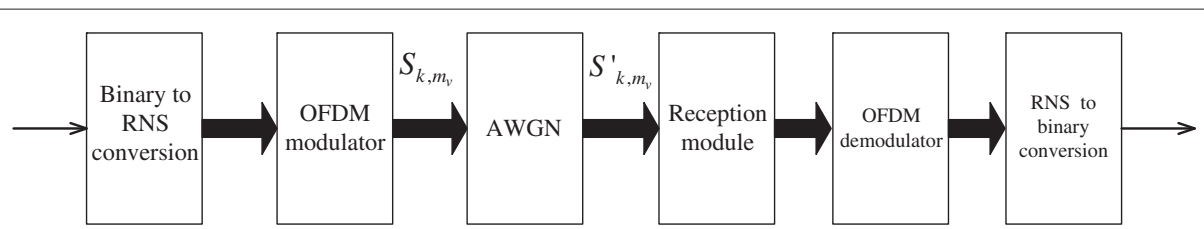

Figure 2 The simplified block diagram of RNS-OFDM.

RNS uniquely and unambiguously, where $M_{I}=\prod_{v=1}^{V} m_{v}$ is referred to as the information dynamic range, i.e., the legitimate range of the information symbol.

For example, given a modulus set $\{7,15,16\}$, the information symbol $R=1,538$, in the information dynamic range $[0,1,680)$, can be presented by $\{5,8,2\}$ in this RNS. In this sense, a big integer can be converted into small residues, which are always smaller than the corresponding moduli.

Usually, the binary-to-residue conversion and the residue-to-binary conversion are denoted as $B / R$ and $R / B$, respectively. The information symbols can be uniquely recovered by residue sequence through CRT [21], which is one of the fundamental theorems of RNS. The relationship between the information symbols $R$ and its residues is as follows (5):

$$
R=\left(\sum_{v=1}^{V} S_{v} \times\left\langle 1 / S_{v}\right\rangle_{m_{v}} \times r_{v}\right) \bmod M_{I}
$$

where $\left\langle 1 / S_{\nu}\right\rangle_{m_{v}}$ is called as multiplicative inverse of $S_{v}$, $S_{v}=M_{I} / m_{v}$, and $\left(S_{v} \times\left\langle 1 / S_{v}\right\rangle_{m_{v}}\right) \bmod m_{v}=1$.

The definition of a signed number in RNS is similar to that in two's complement system (TCS) [13,22], that is, an integer $R$ in the legitimate range $\left[0, M_{I}\right.$ ) can be represented as a signed number, $\widetilde{R}$. Thus, if $0 \leq R<\left\lceil M_{I} / 2\right\rceil$ or $\left\lceil M_{I} / 2\right\rceil \leq R<M_{I}, \widetilde{R}$ is positive or negative, respectively, where $\lceil x\rceil$ denotes the smallest integer larger than $x$.

In RNS, the addition and multiplication are both performed in a modular manner. If the given modulus set is $\left\{m_{1}, m_{2}, \ldots, m_{V}\right\}, X \cong\left\{x_{1}, x_{2}, \ldots, x_{V}\right\}$, and $Y \cong$ $\left\{y_{1}, y_{2}, \ldots, y_{V}\right\}$, i.e., $x_{v}=\langle X\rangle_{m_{v}}$ and $y_{v}=\langle Y\rangle_{m_{v}}$, then the addition/subtraction and multiplication can be presented respectively by (6) and (7):

$$
\begin{aligned}
X \pm Y & \cong\left\{x_{1}, x_{2}, \ldots, x_{V}\right\} \pm\left\{y_{1}, y_{2}, \ldots, y_{V}\right\} \\
& =\left\{\mathrm{z}_{1}, z_{2}, \ldots z_{V}\right\} \cong \mathrm{Z} \text { where } \mathrm{z}_{\mathrm{v}}=\left\langle x_{v} \pm y_{v}\right\rangle_{m_{v}} \\
X \times Y & \cong\left\{x_{1}, x_{2}, \ldots, x_{V}\right\} \times\left\{y_{1}, y_{2}, \ldots, y_{V}\right\} \\
& =\left\{\mathrm{z}_{1}, \mathrm{z}_{2}, \ldots z_{V}\right\} \cong \mathrm{Z} \text { wherez } \\
& =\left\langle x_{v} \times y_{v}\right\rangle_{m_{v}} .
\end{aligned}
$$

In the following section, we propose the RNS-based parallel transmission scheme, which limits the dynamic range of transmitted signals to reduce the PAPR of the system. Also, we will analyze the PAPR performance, complexity performance, and transmission bandwidth with the Shannon theory.

\section{RNS-based parallel transmission}

The simplified baseband block diagram of the RNS-based OFDM transmission scheme (denoted as RNS-OFDM in this paper) is given in Figure 2 (the bold arrow represents for the parallel processing). The original frequency band is divided into $V$ equal portions, and the input signals (i.e., constellation symbols) are converted into $V$ residues by the corresponding modulus set through $B / R$. The frequency-domain symbols in residue form in each residue subchannel are modulated by IFFT to the RNSbased OFDM symbols through the OFDM modulator.

Specifically, Figure 3 portrays the proposed RNS-based parallel transmission scheme designed for OFDM. The number of modulus $\left\{m_{1}, m_{2}, \cdots m_{V}\right\}$ is $V$, and the symbols transmitted are denoted as $d_{0}, d_{1}, d_{2} \cdots, d_{i}, \cdots d_{N-1}$. The function of the mapping module, if the input is positive, can be sent into the $\mathrm{B} / \mathrm{R}$ module directly; otherwise, the input adds the legitimate $M_{I}$ before $\mathrm{B} / \mathrm{R}$.

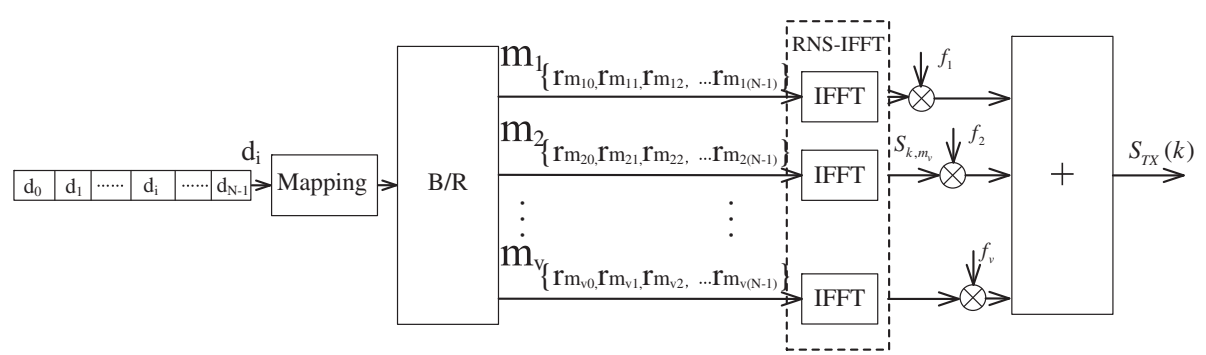

Figure 3 The basic diagram of RNS-based parallel transmission scheme. 
Through $\mathrm{B} / \mathrm{R}$ conversion, according to (4), the serial data streams are divided into $V$ parallel residue subchannels transmitting signals, which are represented as (8) in the RNS.

$$
\left\{\begin{array}{c}
r_{m_{1}, 0}, r_{m_{1}, 1}, \ldots, r_{m_{1},(N-1)} \\
r_{m_{2}, 0}, r_{m_{2}, 1}, \ldots, r_{m_{2},(N-1) ;} \\
\ldots \ldots ; \\
r_{m_{V}, 0}, r_{m_{V}, 1}, \ldots, r_{m_{V},(N-1) ;}
\end{array}\right\}
$$

The residue sequences $\left\{r_{m_{v}, 0}, r_{m_{v}, 1}, \ldots, r_{m_{v},(N-1)}\right\}$ which correspond to the modulus set $m_{v}(v=1,2, \ldots V)$ residue subchannel are transmitted into the IFFT module, respectively. According to (1), the output corresponding to the modulus $m_{v}$ residue subchannel after IFFT is represented as follows (9):

$$
\begin{array}{r}
s_{k, m_{v}}=s(k T / N)=\sum_{i=0}^{N-1} r_{m_{v}, i} \exp \left(j \frac{2 \pi i k}{N}\right) \\
(0 \leq k \leq N-1,0 \leq i \leq N-1) .
\end{array}
$$

The signals of each residue subchannel share the original frequency band through FDM. The set of $V$ parallel residue signals is superimposed in the transmitter expressed as (10)

$$
S_{\mathrm{TX}}(k)=\operatorname{Re}\left[\sum_{v=1}^{V} s_{k, m_{v}} \exp \left(j 2 \pi f_{v}\right)\right],
$$

where $1 \leq v \leq V, f_{v}$ is the carrier frequency.

In the proposed scheme, the parallel transmitting signals of $V$ residue subchannels are simultaneously sent to the channel in $V$ frequency band portions, i.e., assuming these parallel transmitting signals are uncorrelated. Thus, the transmitted signals are superimposed on each other from all frequency bands and are separated on different residue subchannels in FDM. The reception module (in Figure 2) of the receiver is dedicated to receiving signals on the corresponding residue subchannel. Using FFT to demodulate signals for each residue subchannel, the input signals are recovered based on (5) after R/B. The demodulation and detection techniques for receiving RNS-based signals have been proposed in the publications [14,15], which is beyond the scope of this paper.

\subsection{PAPR reduction}

It can be seen from (1) and (2) that the output of OFDM is the sum of the $N$ subcarriers. Wide variation of the amplitudes of OFDM signals could cause high PAPR, which potentially results in nonlinear distortion unless OFDM systems have enough linear dynamic range. When the number of subcarriers is larger, the influence is more critical. However, the dynamic range of the amplifier is usually limited in practical applications. Accordingly, it is better for PAPR reduction that the linear dynamic range of the amplifier is fully used without signal distortion.
The proposed scheme using the properties of RNS effectively reduces the range of transmitted signals. In the following, we will evaluate the PAPR reduction performance by mathematical analysis.

Based on the definition of RNS, the residue is smaller than its corresponding modulus from (4), i.e., $0 \leq r_{v}<$ $m_{v}$,for $1 \leq v \leq V$. The addition/multiplication operation is modular addition/multiplication in RNS, as seen in (6) and (7).We can get (11)

$$
z_{v}=\left\langle r_{m_{v}, 0}+r_{m_{v}, 1}+\ldots+r_{m_{v},(N-1)}\right\rangle_{m_{v}} .
$$

The result is the same in the multiplication in RNS. The output signals after IFFT at the transmitter of this scheme are obtained from (9). Obviously, we can get $z_{v}<m_{v}$, i.e., $\sum_{i=0}^{N-1} r_{m_{v}, i}<m_{v}$.

In other words, regardless of the number of addition and multiplication, the sum of residue signals in each residue subchannel is still smaller than its corresponding modulus. So, this scheme effectively limits the dynamic range of the transmitted signals.

From the definition of the PAPR in (2), the output of OFDM in (1), and the output of RNS-OFDM in (9), the PAPR of OFDM can be presented by (12)

$$
\begin{aligned}
\text { PAPR }_{\text {OFDM }} & =10 \log \frac{\max \left\{\left|\sum_{i=0}^{N-1} d_{i} \exp \left(j \frac{2 \pi i k}{N}\right)\right|^{2}\right\}}{E\left\{\left|\sum_{i=0}^{N-1} d_{i} \exp \left(j \frac{2 \pi i k}{N}\right)\right|^{2}\right\}} \\
& \leq 10 \log \frac{\left|\sum_{i=0}^{N-1} d_{i}\right|^{2}}{E\left\{\left|\sum_{i=0}^{N-1} d_{i} \exp \left(j \frac{2 \pi i k}{N}\right)\right|^{2}\right.}(\mathrm{dB}) .
\end{aligned}
$$

The PAPR of RNS-OFDM also can be obtained as (13)

$$
\begin{aligned}
\mathrm{PAPR}_{\mathrm{RNS}} & =10 \log \frac{\max \left\{\left|\sum_{i=0}^{N-1} r_{m_{v}, i} \exp \left(j \frac{2 \pi i k}{N}\right)\right|^{2}\right\}}{E\left\{\left|\sum_{i=0}^{N-1} r_{m_{v}, i} \exp \left(j \frac{2 \pi i k}{N}\right)\right|^{2}\right\}} \\
& \leq 10 \log \frac{\left.|| \sum_{i=0}^{N-1} r_{m_{v}, i}\right|^{2}}{E\left\{\left|\sum_{i=0}^{N-1} r_{m_{v}, i} \exp \left(j \frac{2 \pi i k}{N}\right)\right|^{2}\right.}(\mathrm{dB})
\end{aligned}
$$

When these phrases of $N$ subcarriers are the same, the highest PAPR occurs, i.e., the inequality sign of the above equation takes an equality sign. In addition, the 
Table 1 Computational complexity of RNS and PTS by the number of equivalent real additions

\begin{tabular}{lll}
\hline PAPR reduction schemes & RNS & PTS \\
\hline Equivalent real additions of IFFT & $21 \mathrm{VN} \log _{2} N$ & $11 \mathrm{MN} \log _{2} N$ \\
Additions of all phrase factors & - & $2(M-1) W^{M-1} N$ \\
Computation of PAPR & $9 V N$ & $9 W^{M-1} N$ \\
Number of equivalent real additions & $21 V N \log _{2} N+9 V N$ & $11 M N \log _{2} N+(2 M+7) W^{M-1} N$ \\
$N=2,048, V=3, W=2, M=8$ & $1,474,560$ & $8,011,776$ \\
\hline
\end{tabular}

real and imaginary parts of the OFDM signal have asymptotically Gaussian distributions for a large number of subcarriers by the central limit theorem. Then, the amplitude of the OFDM signal follows a Rayleigh distribution $R\left(\sigma \sqrt{0.5 \pi},(2-0.5 \pi) \sigma^{2}\right)$, with mean $E(X)=\sigma \sqrt{0.5 \pi}$ and variance $D(X)=(2-0.5 \pi) \sigma^{2}$. From the computational formula for variance [23], we can get (14)

$E\left(X^{2}\right)=D(X)+[E(X)]^{2}=(2-0.5 \pi) \sigma^{2}+0.5 \pi \sigma^{2}=2 \sigma^{2}$.

By substituting (14) to (12) and (13) respectively, we can find that the denominators in the PAPR equation of OFDM and RNS-OFDM are both constant for a specific Rayleigh process. To compare the PAPR performance between OFDM and RNS-OFDM is mainly to compare the fractions, i.e., the size of $\sum_{i=0}^{N-1} d_{i} \exp \left(j \frac{2 \pi i k}{N}\right)$ and $\sum_{i=0}^{N-1} r_{m_{v}, i} \exp \left(j \frac{2 \pi i k}{N}\right)$. According to (4), the residue $r_{m_{v}, i}$ of the number $d_{i}$ is always smaller than $d_{i}$. After multiplying a rotation factor $\exp \left(j \frac{2 \pi i k}{N}\right)$ and summing up all the $N$ elements, we have $\sum_{i=0}^{N-1} r_{m_{v}, i} \exp \left(j \frac{2 \pi i k}{N}\right)<\sum_{i=0}^{N-1} d_{i} \exp \left(j \frac{2 \pi i k}{N}\right)$. Therefore, the PAPR in RNS-OFDM could be much smaller than that in OFDM.

For intuitive description, we show a simple example to compare the PAPR reduction performance between them. The symbols to be transmitted are denoted as $d_{0}, d_{1}, d_{2} \cdots, d_{i}, \cdots d_{N-1}$, where the data $d_{i}$ are selected in this dynamic range $D:[0,128)$, and the number of subcarriers is 1,000 . In conventional OFDM, the output $s_{k}$ gets the high value when the phrases of subcarriers are close to each other. Extremely, if these phrases of all subcarriers equal zero, the output has the highest value from (12). Then, the range of the output is $[0,128,000)$ from (1). In this RNS-OFDM, the modulus set $\{3,7,8\}$ is selected, and the legitimate range $M_{I}$ equals $[0,168)$, where $M \supset D$. In such case, $d_{i}$ can be unambiguously recovered by the residue sequence $\left(r_{1}, r_{2}, r_{3}\right)$. The range of the output of the RNS-OFDM can be evaluated [0, 8, 000) from (9). In the RNS-based parallel transmission scheme, the residue is always smaller than its corresponding modulus, i.e., $0 \leq$ $r_{v}<m_{v}$, for $1 \leq v \leq V$. In addition, the value of each selected modulus can be much smaller than the value of the original data $d_{i}$. As a result, the amplitude of the transmitted signals of the RNS-OFDM decreases more than 10 times as that of the OFDM, and the range of the maximum power reduces more than 100 times.

With respect to of the dynamic range of transmitted signals in the proposed scheme, we have made mathematical analysis and made the preliminary estimate for PAPR reduction performance. The RNS-based parallel transmission scheme for OFDM is expected to improve the PAPR reduction performance. A simulation study will be given in Section 4.

\subsection{Complexity analysis}

We focus our attention on the computational basic units in RNS, modular addition and multiplication, which are the basis of complexity analysis. The computational complexity of the RNS-based scheme will be discussed in this part.

In theoretical analysis, RNS modular addition/multiplication can be designed for flexibility in which case the methodology allows the design of adder/multiplier for any modulus. The basic adder for any modulo- $m$ is defined as (15)

$$
\langle A+B\rangle_{m}= \begin{cases}A+B & \text { if } A+B<\mathrm{m} \\ A+B-m & \text { otherwise. }\end{cases}
$$

The most straightforward implementation, the most complex way, requires three adders: one for the addition, one for the subtraction, and one for the comparison [24]. A modular multiplication of complex signals can be expressed as (16)

$$
\begin{aligned}
\langle A \times B\rangle_{m} & =\left\langle\left\langle a_{1} a_{2}\right\rangle_{m}-\left\langle b_{1} b_{2}\right\rangle_{m}\right\rangle_{m} \\
& +i\left\langle\left\langle b_{1} a_{2}\right\rangle_{m}+\left\langle b_{2} a_{1}\right\rangle_{m}\right\rangle_{m} .
\end{aligned}
$$

The modular multiplier needs more six modular operations than complex multiplier, which needs four real

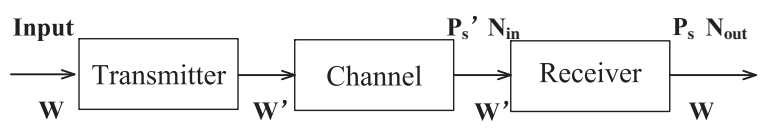

Figure 4 The general diagram of transmission channel. 
Table 2 Parameter used for simulation of PAPR

\begin{tabular}{ll}
\hline Parameters & Value \\
\hline Subcarrier number, $N$ & 2,048 \\
The number of input symbols & 1,000 \\
Modulation format & 64 QAM, 16QAM, QPSK \\
Moduli number, $V$ & 3 \\
Moduli set & $128,127,63$ \\
Phase factors, $W$ & $\{-1,1\}$ \\
Disjoint sub-blocks, $M$ & 3,8 \\
\hline
\end{tabular}

multipliers and two real adders. In each modular operation, it needs two adders (one for addition and one for comparison) in the most straightforward implementation.

In general, a length $N$ IFFT operation requires $(N / 2) \log _{2} N$ complex multiplications and $N \log _{2} N$ complex additions. A complex multiplication takes four real multiplications and two real additions, and a complex addition requires two real additions [8].

In the RNS-based scheme, the number of modulus $V$ $N$-pointed IFFT is needed. Considering the input as the complex signal, a modular complex addition would take 6 real additions, and a modular complex multiplication would take 30 real additions in the high-complexity situation. $\left|s_{k, m_{\nu}}\right|^{2}$ is calculated to determine the PAPR, which requires $2 V N$ real multiplications and $V N$ real additions.

In Table 1, we have summarized the computational complexity of the RNS-based transmission scheme through real addition and compared it with one of the popular lossless PAPR reduction schemes, the PTS [9]. Note that implementation of the RNS-based PAPR reduction scheme is supposed in the most complex way. However, when the binary phase factors of $\{-1,1\}$ are used, i.e., $W=2$, the computational complexity of the rotation of each sub-block for the PTS scheme is reduced. The computational complexity of the RNS-based PAPR reduction scheme is much lower than that of the PTS scheme.

\subsection{Bandwidth analysis}

In the following, we will analyze the transmission bandwidth consumption of the proposed scheme and derive the approximate transmission bandwidth to facilitate the practical application.

From the Shannon formula [25], the channel capacity in unit time is expressed as (17)

$$
C_{t}=\lim _{T \rightarrow \infty} \frac{C}{T}=W \log \left(1+\frac{P_{s}}{n_{0} W}\right)
$$

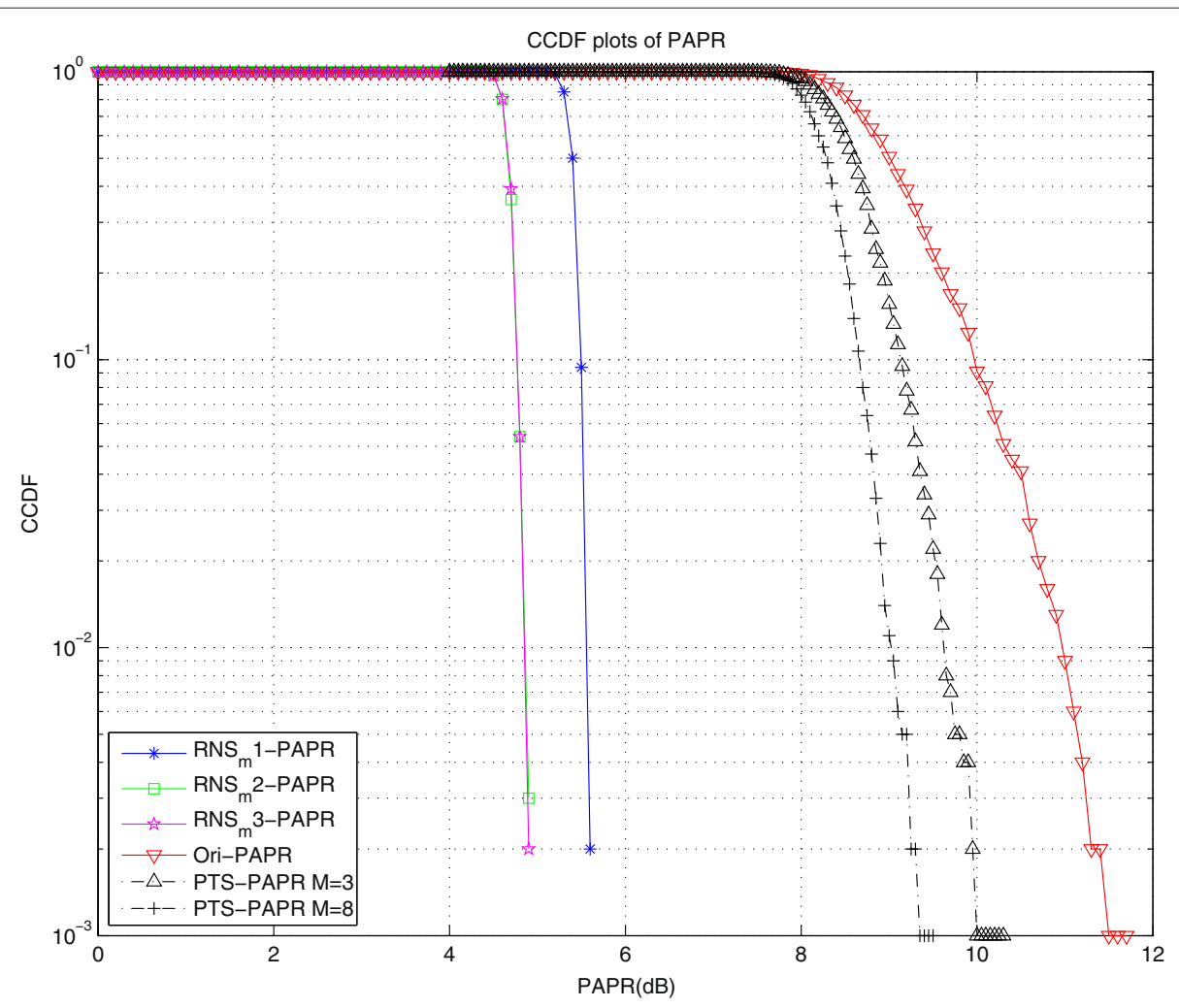

Figure 5 PAPR reduction performance of the proposed scheme, PTS scheme, and the conventional OFDM. With $N=2,048,64 \mathrm{QAM}, \mathrm{V}=3$, $W=\{-1,1\}$, and $M=3 / M=8$. 
where $W$ is the transmission bandwidth, $P_{s}$ is signal power, and $n_{0}$ is white Gaussian noise spectral density. It can be seen that the channel capacity is relevant to the transmission bandwidth and signal-to-noise ratio (SNR).

The general diagram of the transmission channel is shown in Figure 4. When the channel capacity and transmission time are constants, the information rate of input is equal to the information rate of output. Hence, the following relationship is obtained:

$$
W^{\prime} \log \left(1+\frac{P_{s}^{\prime}}{N_{\text {in }}}\right)=W \log \left(1+\frac{P_{s}}{N_{\text {out }}}\right) .
$$

To compare the transmission bandwidth of these two schemes, the RNS-OFDM and conventional OFDM, we derive their relationship expression of transmission bandwidth under the conditions that the input of the transmitters and number of subcarriers of the two schemes are the same. Denote the signal bandwidth as $W_{\text {RNS }}$ (i.e., the transmission bandwidth), signal power as $P_{\text {RNS }}$, and the additive white Gaussian noise channel with signal power

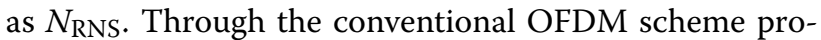
cessing, the transmission bandwidth is denoted as $W$, the signal power as $P_{s}$, and the channel noise power as $N_{0}$. The following expression can be obtained from (18):

$$
W_{\mathrm{RNS}} \log \left(1+\frac{P_{\mathrm{RNS}}}{N_{\mathrm{RNS}}}\right)=W \log \left(1+\frac{P_{s}}{N_{0}}\right) .
$$

When $\frac{P_{s}}{N_{0}}$ is much more than 1, i.e., the signal-to-noise ratio $S N R=10 \log \frac{P_{s}}{N_{0}}$ is larger than $0 \mathrm{~dB}$, the equation above can be facilitated as

$$
\left(\frac{P_{\mathrm{RNS}}}{N_{\mathrm{RNS}}}\right)^{\frac{W_{\mathrm{RNS}}}{W}}=\frac{P_{s}}{N_{0}} .
$$

In order to compare the transmission bandwidth of these two schemes, let $\alpha$ be the coefficient of transmission bandwidth consumption, i.e., $\alpha=W_{\mathrm{RNS}} / W$. So, the coefficient of transmission bandwidth consumption is obtained from (20)

$$
\alpha=\frac{\log \frac{P_{s}}{N_{0}}}{\log \frac{P_{\mathrm{RNS}}}{N_{\mathrm{RNS}}}} .
$$

Since the signal power is $P_{s}=E_{b} \times r$, where $E_{b}$ and $r$ are the energy per information bit and the information transmission rate, respectively, according to the structure of the RNS-based parallel transmission scheme, the relationship of transmission rate between the proposed scheme and the conventional OFDM can be presented by $r_{\mathrm{RNS}}=c \times r$, where the bit-wide coefficient $c=\frac{\log _{2} M_{I}}{\log _{2} M_{\text {ary }}}$, and $M_{\text {ary }}$ represents the $\mathrm{M}$-ary of the input signal. Then, the signal power of RNS-OFDM is obtained as follows:

$$
P_{\mathrm{RNS}}=E_{b} r_{\mathrm{RNS}}=E_{b} c r=c P_{s} .
$$

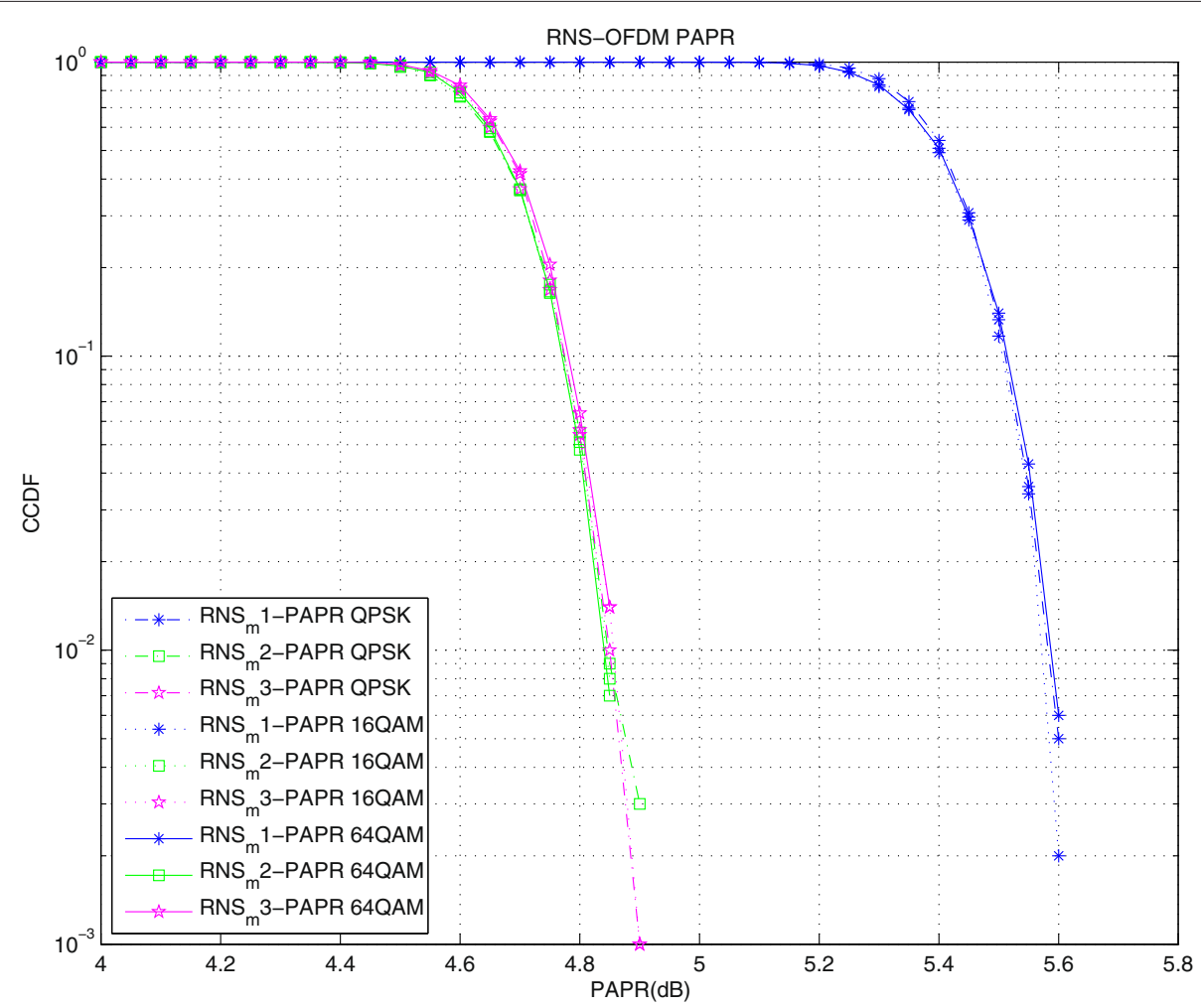

Figure 6 PAPR performance of the RNS-OFDM with 64QAM, 16QAM, and QPSK modulations. 
Since the channel noise average power of OFDM is $N_{0}=$ $n_{0} W$, the noise power of RNS-OFDM can be obtained as follows:

$$
N_{\mathrm{RNS}}=n_{0} W_{\mathrm{RNS}}=n_{0} \alpha W=\alpha N_{0} .
$$

In this case, the following relationship is obtained by substituting (22) and (23) in (21):

$$
\alpha=\frac{\log \frac{P_{s}}{N_{0}}}{\log \frac{c P_{s}}{\alpha N_{0}}}=\frac{\log \frac{P_{s}}{N_{0}}}{\log \frac{c}{\alpha}+\log \frac{P_{s}}{N_{0}}}=\frac{1}{1+\frac{\log c-\log \alpha}{\log \frac{P_{s}}{N_{0}}}} .
$$

In other words, the transmission bandwidth relationship between these two schemes is

$$
W_{\text {RNS }}=\frac{1}{1+\frac{\log c-\log \alpha}{\log \frac{P_{s}}{N_{0}}}} W .
$$

The character of the curve of coefficient of the transmission bandwidth consumption will be shown in the following section, in which $0<\alpha<1$. Obviously, since $\log \alpha<0$ and $\log \frac{P_{s}}{N_{0}}>0$, we can get

$$
0<\alpha=\frac{1}{1+\frac{\log c-\log \alpha}{\log \frac{P_{s}}{N_{0}}}}<\frac{1}{1+\frac{\log c}{\log \frac{P_{s}}{N_{0}}}}<1 .
$$

To facilitate the practical application, the approximate formula of the transmission bandwidth between the proposed scheme and conventional one, as seen in (27), is obtained after simplifying (25). The verification and analysis of the approximate formula will be shown in the following section

$$
W_{\mathrm{RNS}}=\frac{1}{1+\frac{\log c}{\log \frac{P_{s}}{N_{0}}}} W
$$

The transmission bandwidth of this scheme is relevant to the bit-wide coefficient $c$ and SNR. When $c=1$, i.e., the dynamic range equals the value of $\mathrm{M}$-ary of the input signal, is met, then both transmission bandwidths are equal. When the condition $\frac{P_{s}}{N_{0}}>1$, i.e., SNR $>$ $0 \mathrm{~dB}$ and $c>1$, is met (in general, SNR is bigger than $0 \mathrm{~dB}$ in practical applications), the transmission bandwidth of the proposed scheme is smaller than the one of OFDM. This could be explained that when $c>1$, the total number of bits per residue symbol is also bigger than the number of bits of the $\mathrm{M}$-ary input, implying that the energy per symbol increases. Hence, the bandwidth is decreased.

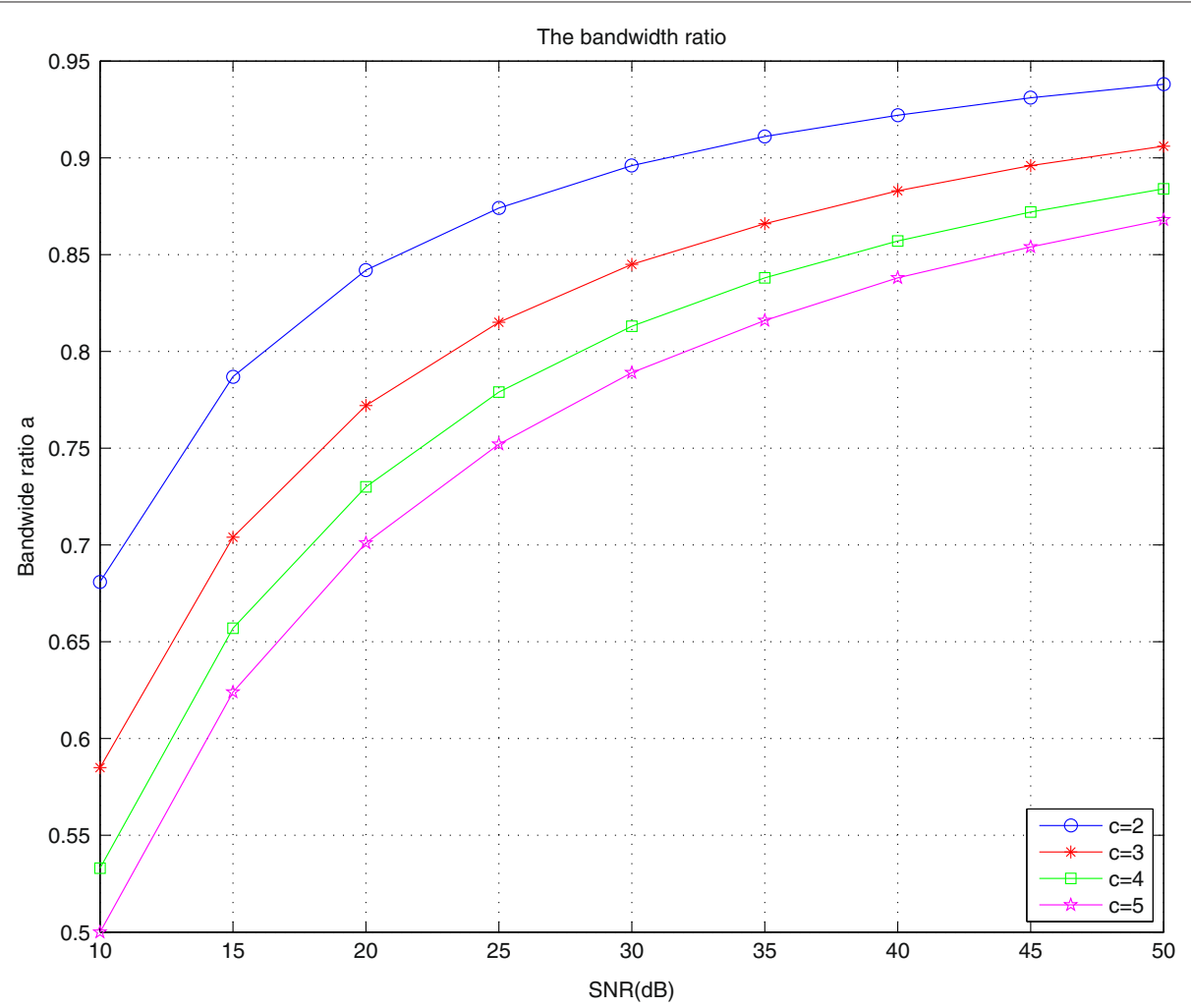

Figure 7 The relationship between the bandwidth consumption ratio and SNR with different bit-wide coefficients $c$. 
Table 3 Approximate results of bandwidth consumption

\begin{tabular}{llllll}
\hline $\boldsymbol{c}$ & \multicolumn{3}{c}{ SNR } & & \\
\cline { 2 - 6 } & $\mathbf{1 0} \mathbf{d B}$ & $\mathbf{2 0} \mathbf{d B}$ & $\mathbf{3 0 ~ d B}$ & $\mathbf{4 0} \mathbf{d B}$ & \\
\hline 2 & $76.9 \%$ & $86.9 \%$ & $90.9 \%$ & $93.0 \%$ & \\
3 & $67.7 \%$ & $80.7 \%$ & $86.3 \%$ & $89.3 \%$ & $94.3 \%$ \\
4 & $62.4 \%$ & $76.9 \%$ & $83.3 \%$ & $86.9 \%$ & $89.3 \%$ \\
5 & $58.9 \%$ & $74.1 \%$ & $81.1 \%$ & $85.1 \%$ & $87.7 \%$ \\
\hline
\end{tabular}

\section{Simulation results}

We use simulations to study PAPR reduction, transmission bandwidth performance, and the out-of-band (OOB) spectrum of the proposed scheme and to evaluate the complexity performance through the comparison of 2048FFT between the RNS-FFT and TCS-FFT.

The performance of PAPR reduction is evaluated by $\mathrm{CCDF}$. The simulations assumed that the number of modulus $V$ was equal to 3 and the value of the modulus set $\left\{m_{1}, m_{2}, m_{3}\right\}$ was $\{128,127,63\}$. The parameter used for simulation is shown in Table 2.

Figure 5 compares the PAPR reduction performance of the proposed scheme, the PTS scheme, and the conventional OFDM, where the modulation style is 64QAM. The curves labeled by 'RNS $m_{1}$ - PAPR', 'RNS_ $m_{2}-$ PAPR', and 'RNS_ $m_{3}-$ PAPR' denote the PAPR performance of the residue subchannels, respectively, which correspond to the modulus set $\left\{m_{1}, m_{2}, m_{3}\right\}$. The curves labeled by 'PTS-PAPR $M=3$ ' and 'PTS-PAPR $M=8$ ' denote the PAPR performance of the PTS scheme with $M=3$ and $M=8$ disjoint sub-blocks, respectively. When the PTS is with $M=3, W=\{-1,1\}$, the RNS-based scheme is better than the PTS by about $4 \mathrm{~dB}$. When the PTS is with $M=8$, the proposed scheme still outperforms PTS. Meanwhile, the computational complexity of the RNSbased scheme is less than $20 \%$ of that of the PTS. The curve labeled by 'Ori-PAPR' denotes the conventional OFDM PAPR performance. More than 5-dB improvement of PAPR reduction is obtained by the RNS-based parallel transmission scheme at a CCDF of $10^{-2}$.

The PAPR reduction performance of the proposed scheme is simulated with different modulation styles, viz.

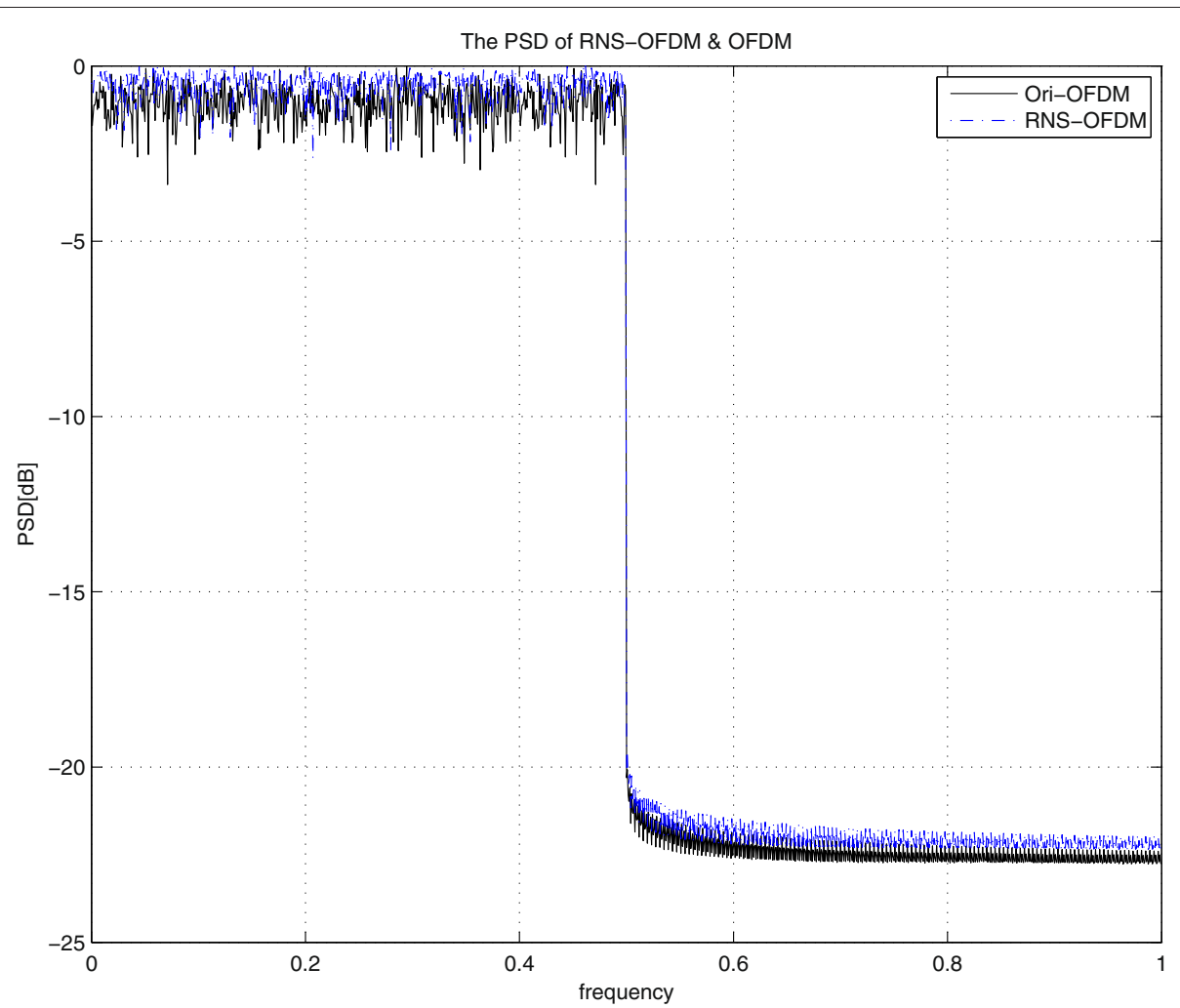

Figure 8 The power spectral density of RNS-OFDM and OFDM with $N=2,048,64$ QAM. 
Table 4 Complexity performance of RNS-FFT and TCS-FFT for 2048-point FFT

\begin{tabular}{lll}
\hline & RNS-FFT & TCS-FFT \\
\hline Combinational ALUT (used/total) & $9,925 / 182,400(5 \%)$ & $13,294 / 182,400(7 \%)$ \\
Memory ALUT (used/total) & $162 / 91,200(<1 \%)$ & $0 / 91,200(0 \%)$ \\
Registers (used/total) & $3,101 / 182,400(2 \%)$ & $1,361 / 182,400(<1 \%)$ \\
Frequency of critical path (MHz) & 114.08 & 106.09 \\
\hline
\end{tabular}

ALUT, adaptive look-up table.

64QAM, 16QAM, and QPSK, as shown in Figure 6. These curves denote the PAPR performance of the residue subchannels with different modulation styles, respectively. While the modulation style changes, the PAPR performance curves of each residue subchannel scarcely change. The results show that the proposed scheme can effectively reduce the PAPR compared with the conventional transmission scheme. At the same time, the proposed scheme is not restricted to any modulation format in any residue subchannel.

The relationship curves between $\alpha$ and SNR based on (25), as shown in Figure 7, portray the transmission bandwidth consumption ratio curves with different bit-wide coefficients $c$, which vary from 2 to 5 . For instance, when the coefficient $c=3$ and the signal-to-noise ratio SNR = $20 \mathrm{~dB}$, the transmission bandwidth consumption of the RNS-OFDM is reduced to $77.2 \%$ of that of conventional OFDM.

The results in Table 3 present the bandwidth ratio between the RNS-OFDM and the conventional one, which are obtained from (27) with bit-wide coefficients $c$ and SNR. Compared to the bandwidth ratio curve in Figure 7, the approximate results in Table 3 are a little larger than the real results, but the approximate formula facilitating the calculation is very useful in practical application. Furthermore, according to the analysis above, the proposed scheme occupies less bandwidth resource than the conventional one. As a general trend, the transmission bandwidth changes exponentially with different SNRs, as seen in (20); thereby, we can adjust SNR through the minor adjustment of the number of modulus, or the dynamic range of RNS, to get a comparatively better improvement of the transmission bandwidth consumption.

The OOB spectrum can be observed from Figure 8 . The spectrums of RNS-OFDM and OFDM are almost the same. This could be explained by the computational process of PSD which is equivalent to the conventional FFT of the signal envelope.

Regarding the hardware complexity, it can be seen that when the proposed scheme increases the additional R/B and the residue subchannels units, the hardware complexity of this scheme rises. However, at the same time, it completely preserves the advantages of RNS, such as parallelism, carry-free, high speed, etc. We illustrate the complexity performances of the proposed RNS-based FFT by comparing it with the TCS-based FFT in FPGA. As shown in Table 4, our implementation is comparable to TCS-FFT in terms of hardware complexity, but it outperforms TCS-FFT with respect to computation speed. In practical hardware implementation, the RNS-based parallel transmission scheme possibly would seek a tradeoff between the hardware complexity and computation speed.

\section{Conclusions}

This paper has presented a novel PAPR reduction scheme, where the natural properties of RNS are utilized to ensure that the amplitude of the transmitted signals has a smaller range for PAPR reduction. Simulation results demonstrate that the proposed scheme achieves 5-dB PAPR improvement compared with the conventional OFDM and also outperforms PTS with low computational complexity. A bandwidth approximate formula of the proposed scheme is derived, which simplifies the design procedure in practice. Though the hardware complexity of the proposed scheme rises, the proposed scheme would seek a tradeoff between the hardware complexity and computation speed.

\section{Competing interests}

The authors declare that they have no competing interests.

\section{Authors' information}

YY is a Ph.D student of the National Key Lab of Science and Technology on Communication, University of Electronic Science and Technology of China (UESTC), Chengdu 611731, China. JH is a full professor of the National Key Lab of Science and Technology on Communication, UESTC, Chengdu 611731, China. JH is also an IEEE member. SM is an associate professor of the National Key Lab of Science and Technology on Communication, UESTC, Chengdu 611731, China.

\section{Acknowledgements}

This work was supported in part by the National Natural Science Foundation of China under grants 61076096 and 61101033 and by the Fundamental Research Funds for the Central Universities under grant ZYGX2011 J118.

Received: 24 October 2012 Accepted: 19 May 2013

Published: 7 June 2013 


\section{References}

1. L Dai, Z Wang, Z Yang, Time-frequency training OFDM with high spectral efficiency and reliable performance in high speed environments. IEEE J. Sel. Areas Commun. 30(4), 695-707 (2012)

2. L Dai, Z Wang, J Wang, Z Yang, Positioning with OFDM signals for the next-generation GNSS. IEEE Trans. Consumer Electron. 56(2), 374-379 (2010)

3. T Jiang, Y Wu, An overview: peak-to-average power ratio reduction techniques for OFDM signals. Broadcasting, IEEE Trans. 54(2), 257-268 (2008)

4. SH Han, JH Lee, An overview of peak-to-average power ratio reduction techniques for multicarrier transmission. Wireless Commun. IEEE. 12(2), 56-65 (2005)

5. Y Xiao, X Lei, S Li, in International Conference on Communications, Circuits and Systems, 2009. ICCCAS 2009. (2009). Modified clipping and filtering scheme for OFDM systems (IEEE Milpitas, 2009), pp. 38-42

6. F Juwono, D Gunawan, in Innovative Technologies in Intelligent Systems and Industrial Applications, 2009. CITISIA 2009. PAPR reduction using Huffman coding combined with clipping and filtering for OFDM transmitter (IEEE Monash, 2009), pp. 344-347

7. F Juwono, D Gunawan, in Wireless Communications Networking and Mobile Computing (WiCOM), 2010 6th International Conference on. The effectiveness of using source coding to reduce PAPR in OFDM system (IEEE Chengdu, 2010), pp. 1-3

8. Q Wen, Y Xiao, P Cheng, L Dan, S Li, in Vehicular Technology Conference, 2008. VTC 2008-Fall. IEEE 68th. A modified partial transmit sequence scheme for PAPR reduction in OFDM system (IEEE Calgary, 2008), pp. 1-5

9. R Baxley, G Zhou, Comparing selected mapping and partial transmit sequence for PAR reduction. Broadcasting, IEEE Trans. 53(4), 797-803 (2007)

10. T Kojima, Y Shida, T Fujino, in 2010 International Conference on Advanced Technologies for Communications (ATC). A study of SLM PAPR reduction of OFDM signals without side information (IEEE Ho Chi Minh City, 2010), pp. 168-171

11. $Y Y e, J H u, S M a, X L i n g$, in International Conference on Communications, Circuits and Systems, 2009. ICCCAS 2009. A new efficient RNS-to-binary conversion for the three-moduli sets (IEEE Milpitas, 2009), pp. 1000-1005

12. $\mathrm{S} M a, J \mathrm{Hu}, \mathrm{L}$ Zhang, X Ling, An efficient RNS parity checker for moduli set $\{2 n-1,2 n+1,22 n+1\}$ and its applications. Sci. China Series F: Inf. Sci. 51(10), 1563-1571 (2008)

13. $S \mathrm{Ma}, \mathrm{JHu}, \mathrm{Y} Y \mathrm{Y}$, L Z hang, $X$ Ling, A $2 \mathrm{n}$ scaling scheme for signed RNS integers and its VLSI implementation. SCI. CHINA Inf Sci. 53, 203-212 (2010)

14. LL Yang, L Hanzo, A residue number system based parallel communication scheme using orthogonal signaling .I. System outline. Vehicular Technol, IEEE Trans. 51(6), 1534-1546 (2002)

15. LL Yang, L Hanzo, A residue number system based parallel communication scheme using orthogonal signaling. II. Multipath fading channels. Vehicular Tech. IEEE Trans. 51(6), 1547-1559 (2002)

16. D Zhu, B Natarajan, in Vehicular Technology Conference Fall (VTC 2009-Fall), 2009 IEEE 70th. Residue number system arithmetic aided frequency-hopping pattern design in coded OFDMA (IEEE, 2009), pp. 1-5

17. Z Dalin, N Balasubramaniam, Residue number system arithmetic assisted coded frequency-hopped OFDMA. EURASIP J. Wireless Commun. Net. 2009 (2009). doi:10.1155/2009/263695

18. D Zhu, B Natarajan, Residue number system arithmetic-inspired hopping-pilot pattern design. Vehicular Tech. IEEE Trans. 59(7), 3679-3683 (2010)

19. $\mathrm{Y} Y \mathrm{i}, \mathrm{H}$ Jian-hao, in 2011 International Conference on Computational Problem-Solving (ICCP). RNS based OFDM transmission scheme with low PAPR (IEEE Chengdu, 2011), pp. 326-329

20. J Palicot, Y Louët, in European Signal Processing Conference, EUSIPCO. Power ratio definitions and analysis in single carrier modulations (Antalya, Turkey, 4-8 September 2005)

21. Y Wang, in Signals, Systems amp; Computers, 1998. Conference Record of the Thirty-Second Asilomar Conference on, vol. 1. New Chinese remainder theorems (IEEE Pacific Grove, 1998), pp. 165-171

22. H Garner, The residue number system. Electron Comput, IRE Trans. EC-8(2), 140-147 (1959)
23. L Tierney, J Kadane, Accurate approximations for posterior moments and marginal densities. J. Am. Stat. Assoc. 81(393), 82-86 (1986)

24. A Omondi, B Premkumar, Residue Number Systems: Theory and Implementation. (Imperial College Press, London, 2007)

25. C Shannon, W Weaver, R Blahut, B Hajek, The Mathematical Theory of Communication, vol. 117. (University of Illinois Press, Urbana, 1949)

doi:10.1186/1687-1499-2013-156

Cite this article as: Yao et al:: A PAPR reduction scheme with residue number system for OFDM. EURASIP Journal on Wireless Communications and Networking 2013 2013:156.

\section{Submit your manuscript to a SpringerOpen ${ }^{\circ}$ journal and benefit from:}

- Convenient online submission

Rigorous peer review

- Immediate publication on acceptance

- Open access: articles freely available online

- High visibility within the field

- Retaining the copyright to your article

Submit your next manuscript at $>$ springeropen.com 